\title{
High speed logic gate using two-photon absorption in silicon waveguides
}

\author{
T.K. Liang ${ }^{\mathrm{a}, *}$, L.R. Nunes ${ }^{\mathrm{a}}$, M. Tsuchiya ${ }^{\mathrm{a}}$, K.S. Abedin ${ }^{\mathrm{b}}$, T. Miyazaki ${ }^{\mathrm{b}}$, \\ D. Van Thourhout ${ }^{c}$, W. Bogaerts ${ }^{\text {c }}$, P. Dumon ${ }^{c}$, R. Baets ${ }^{c}$, H.K. Tsang ${ }^{d}$ \\ a Basic and Advanced Research Department, National Institute of Information and Communications Technology, 4-2-1 Nukui-Kitamachi, Koganei, \\ Tokyo 1848795, Japan \\ ${ }^{\mathrm{b}}$ Information and Network Systems Department, National Institute of Information and Communications Technology, Koganei, Tokyo, Japan \\ ${ }^{\mathrm{c}}$ Department of Information Technology, University of Ghent, Ghent, Belgium \\ d Department of Electronic Engineering, The Chinese University of Hong Kong, Shatin, Hong Kong
}

Received 23 January 2006; received in revised form 3 March 2006; accepted 7 March 2006

\begin{abstract}
The switching speed of conventional silicon-based optical switching devices based on plasma dispersion effect is limited by the lifetime of free carriers which introduce either phase or absorption changes. Here we report an all-optical logic NOR gate which does not rely on free carriers but instead uses two-photon absorption. High speed operation was achieved using pump induced non-degenerate two-photon absorption inside the submicron size silicon wire waveguides. The device required low pulse energy (few pJ) for logic gate operation.
\end{abstract} (C) 2006 Elsevier B.V. All rights reserved.

All-optical digital signal processing may be needed in future high capacity optical networks to overcome the speed limitations of electronics. All-optical logic gates such as NOR gate will be needed to perform the optical digital signal processing to accommodate the massive amount of traffic in terabit optical networks [1]. In addition, NOR gate can be used in performance monitoring for error detection, address and header recognition [2], encryption and data encoding [3], etc. Optical logic gates have been demonstrated in nonlinear optical fibers [4], semiconductor optical amplifier (SOA) [5] and InGaAs/AlAsSb coupled double-quantum-well structures [6]. The latency and high optical power level for nonlinear operation make fiberbased devices unattractive for practical applications, while the long carrier lifetime in conventional SOAs may limit the speed unless some complicated differential switching scheme is employed.

Submicron size silicon wire waveguides are possible because of the extremely high index contrast $(n=3.5$ for

\footnotetext{
${ }^{*}$ Corresponding author. Tel.: +81 423276125; fax: +81423275328

E-mail address: liang.tk@gmail.com (T.K. Liang).
}

silicon, and $n=1.45$ for $\mathrm{SiO}_{2}$ ), which allows the dimension of waveguides to be much smaller than in conventional low index contrast silica waveguidess [7]. The strong optical confinement and small effective modal area $\left(<0.1 \mu \mathrm{m}^{2}\right)$ of such waveguides can produce high optical intensities even at input optical powers typically used in telecommunications. The high optical intensities and long interaction lengths in the waveguides allow nonlinear optical effects to be readily apparent. Apart from other nonlinear optical devices such as optical fibers and SOA, silicon wire waveguides have good potential for other nonlinear devices for ultrafast photonics signal processing [8].

We demonstrated previously an ultrafast optical switch $(<3 \mathrm{ps})$ with $\mathrm{pJ}$ pump pulse energy in wire waveguides $[9,10]$. In this paper, we develop a high speed all-optical logic NOR gate based on the nonlinear transmission characteristics of two-photon absorption (TPA) in silicon. The direct use of TPA allows operation speeds which are not limited by the slow photo-generated carrier lifetime in the silicon wire waveguides.

Since the sum of energies of two photons at $1.55 \mu \mathrm{m}$ wavelength is greater than the indirect bandgap of silicon, 
high intensity pulses will experience phonon assisted TPA when propagating along the waveguide. The amount of absorption is proportional to the square of intensity and the maximum transmitted power is limited. The absorption of photons will lead to two direct consequences - the optical power depletion (photon absorption) and the generation of excess electron-hole pairs (free carriers). The former is intrinsically an ultrafast process [11], while the latter is a slow process that will further attenuate the optical signal via free-carrier absorption and hot carrier assisted absorption. If there are two light beams with slightly different energies (or wavelengths), with one source at high peak power (pump) and the other one at low power (probe), the high power pump source will then induce absorption of the low power probe signal.

A preliminary two-color time-resolved pump-probe experiment was performed to measure the nonlinear transmission of weak probe pulses in the presence of strong optical pump pulses in silicon wire waveguides. The fabrication and characterization of the waveguide was described elsewhere [12]. As shown in the inset of Fig. 1, the waveguide core was formed by a silicon stripe measuring $480 \mathrm{~nm}$ by $220 \mathrm{~nm}$. The length of the waveguide used was $10 \mathrm{~mm}$. The measured transmission as a function of peak pump pulse levels is shown in Fig. 1. Both pump and probe pulses were generated by the spectral slicing of a broadband femtosecond passive mode-locked laser. By using ultrashort pulses (around 1.6 ps FWHM pulsewidth), the additional absorption loss due to photo-generated free carriers was negligible.

Since ultrashort pulses were used to achieve high peak power and low average power, the amount of free carriers generated was small. Assuming the pump pulses have the Gaussian temporal profile, the carrier density created from a single pump pulse inside the waveguide along propagation direction $z$ is denoted by [13]

$N(z)=\frac{\beta \sqrt{\pi} T I_{0}^{2}(z)}{4 h v}$

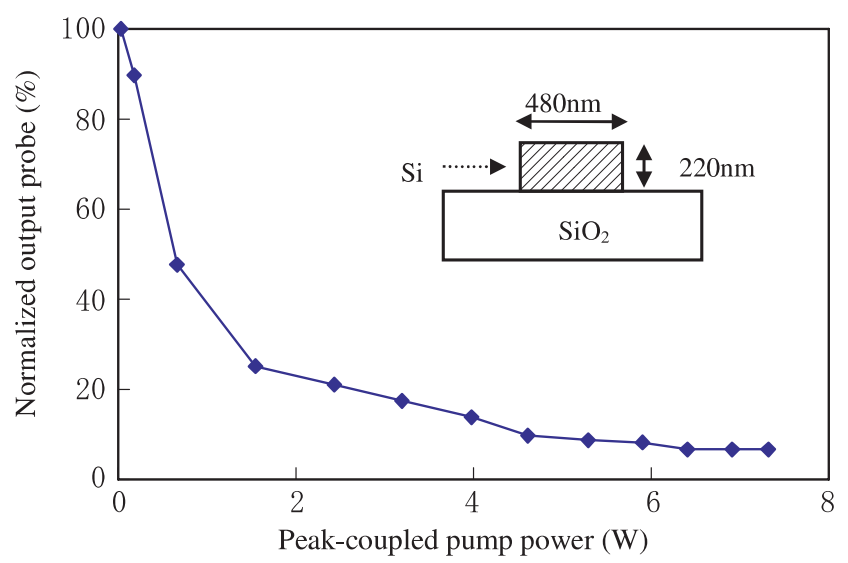

Fig. 1. Measured probe transmission as a function of peak pump pulse levels. Inset: waveguide cross section.

where $\beta$ is the TPA coefficient in silicon, $T$ is the pulsewidth, $I_{0}$ is the peak power and $h v$ is the photon energy. The pump depletion can be described by

$\frac{\mathrm{d} I}{\mathrm{~d} z}=-\alpha I-\beta I^{2}-\alpha_{\mathrm{f}} I$

where $I$ is the intensity of the pulse, $\alpha$ and $\alpha_{\mathrm{f}}$ are the linear propagation loss and free-carrier absorption loss, respectively. The magnitude of free-carrier absorption depends on the carrier density through the relation [11]

$\alpha_{\mathrm{f}}(z)=1.45 \times 10^{-17} N(z)$

For Gaussian pump pulse with 1.6 ps pulsewidth and $2 \mathrm{~W}$ peak power, the calculated free-carrier absorption loss after $1-\mathrm{cm}$ long waveguide will be less than $0.18 \mathrm{~dB}$. Thus the additional loss from photo-generated carriers is almost negligible, which can also be evidenced by the absence of a slow carrier recovery process in the experimental results. The coupled-peak power of probe pulse in the experiment was less than $20 \mathrm{~mW}$. As shown in the curve, the probe pulse was extinguished by more than $90 \%$ at peak-coupled pump power of $5 \mathrm{~W}$. The nonlinear transmission characteristic was due to the pump depletion in the waveguide.

Fig. 2(c) shows the schematic diagram of the optical NOR gate. Signals P1 and P2 are coupled together and used as the pump beam, while a continuous-wave $(\mathrm{CW})$ light is used as the probe beam. As long as one of the two signals is bit " 1 " (includes " 01 ", " 10 ", and " 11 "), the pump beam induces optical absorption on the probe beam in silicon waveguides by means of non-generate TPA effect. By carefully selecting the pump power level, all above cases will induce same loss on probe beam. Once P1 and P2 are both bit " 0 ", the probe beam transmits through the waveguide without additional nonlinear loss. Therefore, Boolean NOR operation can be achieved. The corresponding truth table is shown in Fig. 2(b). The operation principle of

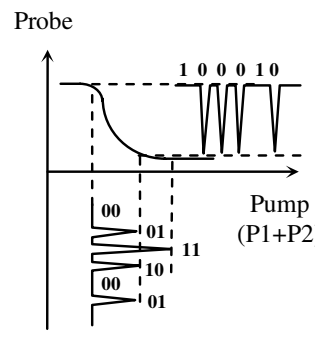

(a)

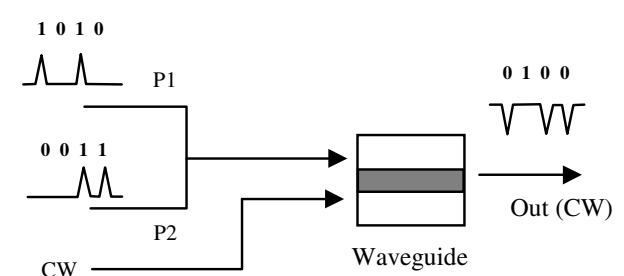

(c)

\begin{tabular}{|c|c|c|}
\multicolumn{3}{c}{ NOR } \\
\hline P1 & P2 & Out \\
\hline 0 & 0 & 1 \\
\hline 0 & 1 & 0 \\
\hline 1 & 0 & 0 \\
\hline 1 & 1 & 0 \\
\hline
\end{tabular}

(b)
Fig. 2. (a) Operation principle. (b) Truth table. (c) Schematic diagram of NOR gate. 
NOR gate based on the nonlinear transmission curve in silicon wire waveguides is shown in Fig. 2(a). Signals P1 and P2 with same peak power were combined together and coupled into the waveguide. The weak $\mathrm{CW}$ probe light at waveguide output was cross-modulated by the sum of $\mathrm{P} 1$ and $\mathrm{P} 2$ based on non-degenerate TPA process. The Boolean NOR operation was achieved in the form of dark pulses. It is worth mentioning that if the $\mathrm{CW}$ probe light is replaced by the pulse probe, then the output of this logic NOR gate can be used as the pump source for the following stages. This cascade ability of the optical logic gate is essential for the real application in designing the complicate logic systems.

The experimental setup for the demonstrating the optical NOR gate is shown in Fig. 3. A stretched pulse passively mode-locked fiber laser (MLFL) was used to generate femtosecond optical pulses at $20 \mathrm{MHz}$ repetition rate. The pulse source, with $50 \mathrm{~nm}$ spectral width, was then split into two paths and passed through two optical tunable filters with center wavelengths at $1545 \mathrm{~nm}$ and $1555 \mathrm{~nm}$, respectively. Part of the passively mode-locked pulses was tapped out to trigger the sampling oscilloscope. The pulses were multiplexed with 25 ps and 12.5 ps delay lines to produce a pulsetrain at 80 Gbps equivalent data rate. The pulse packet was used as signals P1 and P2, respectively. Signal P1 carried the digital signal " 1010 ", while signal P2 carried the signal "0011". A weak CW probe signal, generated by a tunable laser (TL) at $1560 \mathrm{~nm}$, was launched into the silicon wire waveguide together with $\mathrm{P} 1$ and $\mathrm{P} 2$. The tunable filter after the waveguide removed both signals. Finally, the $\mathrm{CW}$ probe formed output logic signal and was detected by a $50 \mathrm{GHz}$ bandwidth photodiode.

The pulse packets $\mathrm{P} 1$ and $\mathrm{P} 2$ are shown in Fig. 4(a) and (b), respectively. The pulsewidth of the signals after TF1 and TF2 were both measured to be 1.6 ps FWHM by an autocorrelator. The measured pulses on the sample oscilloscope were broadened to around 13 ps due to the limited bandwidth of the photodetector. The pulse packets were selected to be at different wavelengths to avoid interference

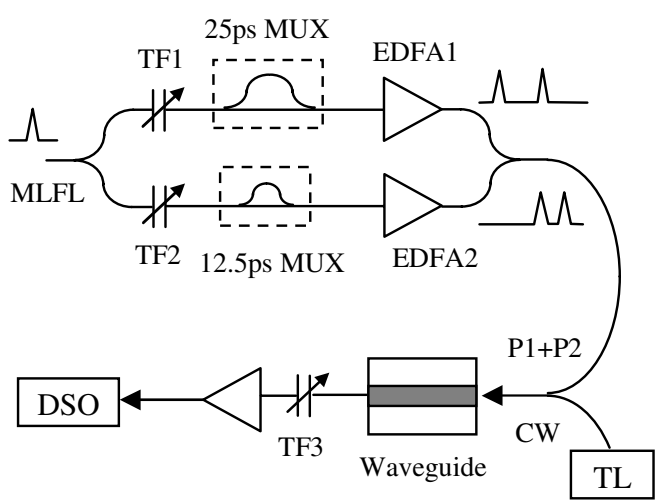

Fig. 3. Experimental setup of all-optical logic NOR gate. MLFL: modelocked fiber laser. TF: tunable filter, MUX: multiplexer, EDFA: erbiumdoped fiber amplifier, TL: tunable laser and DSO: digital sampling oscilloscope. (a)

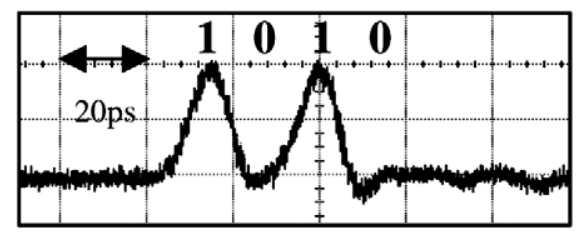

(b)

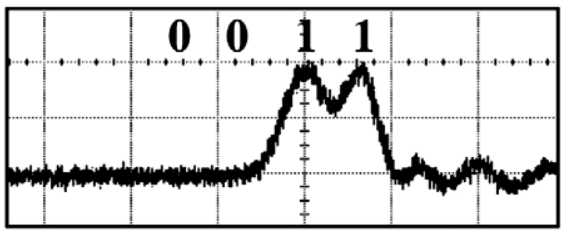

(c)

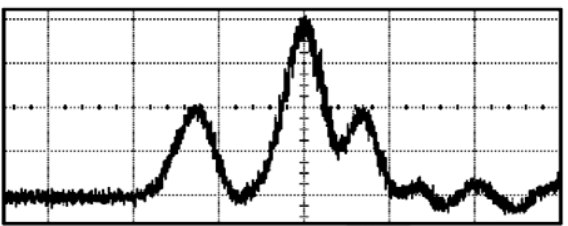

(d)

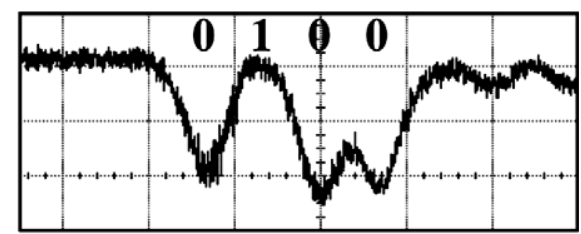

Fig. 4. (a) Signal P1. (b) Signal P2. (c) Combined P1 and P2, corresponding to digital " 10 ", " 00 ", " 11 ", and " 01 ". (d) Output crossmodulated $\mathrm{CW}$ probe with logic NOR operation.

such that a stable output waveform was produced. The peak powers of $\mathrm{P} 1$ and $\mathrm{P} 2$ were less than $5 \mathrm{~W}$. Thus the corresponding pulse energies were less than $8 \mathrm{pJ}$. The actual modulation depth measured by the oscilloscope is limited by the bandwidth of the photodetector. Based on the other individual time-resolved pump-probe measurement, the modulation depth of output dark pulse at this pump power level is expected to be more than $90 \%$. The combined signals $\mathrm{P} 1$ and $\mathrm{P} 2$ before the waveguide are shown in Fig. 4(c). It is apparent from Fig. 4(d) that the output logic NOR operation was " 0100 ". The primary advantage of the scheme is that the logic output can be obtained at any arbitrary wavelength range below the bandgap of silicon. However, additional investigation on the potential limitation of our scheme is required in future. Instead of using 4 bit data, the accumulation of free carriers will possibly become a limiting factor on the maximum operation speed if the real pseudorandom data sequences are used. Other individual experiments showed that the device can operate at more than $10 \mathrm{~Gb} / \mathrm{s}$ real data. The maximum working speed will depend on the pulse width used. The shorter pulse width leads to less carrier accumulation, thus higher speed will be achievable.

In conclusion, a high speed optical logic NOR gate using silicon wire waveguide was demonstrated. The device consisted of only a single 1-cm long waveguide. Only low pump pulse energy was required and operation at any wavelength between $1200 \mathrm{~nm}$ and beyond $1700 \mathrm{~nm}$ range is possible provided the sum of pump photon energy and signal photon energy is larger that the bandgap of silicon. 


\section{Acknowledgement}

This work was funded in part by CUHK RGC Grant 415905.

\section{References}

[1] S.A. Hamilton, B.S. Robinson, T.E. Murphy, S.J. Savage, E.P. Ippen, J. Lightwave Technol. 20 (2002) 2086.

[2] T. Fjelde, A. Kloch, D. Wolfson, B. Dagens, A. Coquelin, I. Guillemot, F. Gaborit, F. Poingt, M. Renaud, Proceedings of ECOC 2000, Munich, Germany, vol. 4, 2000, p. 63.

[3] T. Houbavlis, K. Zoiros, A. Hatziefremidis, H. Avramopoulos, L. Occhi, G. Guekos, S. Hansmann, H. Burkhard, R. Dall Ara, Electron. Lett. 35 (1999) 1650.

[4] M.N. Islam, Opt. Lett. 15 (1990) 417.

[5] A. Hamie, A. Sharaiha, M. Guegan, B. Pucel, IEEE Photon. Technol. Lett. 14 (2002) 1439.
[6] M. Naruse, H. Yoshida, T. Miyazaki, F. Kubota, H. Ishikawa, IEEE Photon. Technol. Lett. 17 (2005) 1701.

[7] R.L. Espinola, J.I. Dadap, R.M. Osgood Jr., S.J. McNab, Y.A Vlasov, Opt. Express 12 (16) (2004) 3713.

[8] T. Kamiya, M. Tsuchiya, Jpn. J. Appl. Phys. Part 1 Rev. Papers 44 (2005) 5875 .

[9] T.K. Liang, L.R. Nunes, T. Sakamoto, K. Sasagawa, T. Kawanishi, M. Tsuchiya, G.R.A. Priem, D. Van Thourhout, P. Dumon, R. Baets, H.K. Tsang, Opt. Express 13 (2005) 7298.

[10] L.R. Nunes, T.K. Liang, K.S. Abedin, D. Van Thourhout, P. Dumon, R. Baets, H.K. Tsang, T. Miyazaki and M. Tsuchiya, in: Proceedings of European Conference on Optical Communication, PDP-4.2.3, Glasgow, Scotland, September 2005.

[11] H.K. Tsang, R.V. Penty, I.H. White, R.S. Grant, W. Sibbett, J.B.D Soole, H.P. Leblanc, N.C. Andreadakis, R. Bhat, M.A. Koza, J. Appl. Phys. 70 (1991) 3992.

[12] W. Bogaerts, D. Taillaert, B. Luyssaert, P. Dumon, J. Van Campenhout, P. Bienstman, D. Van Thourhout, R. Baets, V. Wiaux, S. Beckx, Opt. Express 12 (2004) 1583.

[13] F.R. Laughton, J.H. Marsh, Appl. Phys. Lett. 60 (1992) 166. 